\title{
Efecto del almacenamiento de bagazo de caña en las propiedades físicas de celulosa grado papel
}

\author{
Effect of Storing of Sugar Cane Bagasse on Physical Properties \\ from Cellulose for Paper \\ Aguilar-Rivera N. \\ Facultad de Ciencias Biológicas y Agropecuarias \\ Universidad Veracruzana \\ E-mail:naguilar@uv.mx
}

Información del artículo: recibido: septiembre de 2008, reevaluado: marzo de 2010, aceptado: septiembre de 2010

\begin{abstract}
Resumen
El bagazo de caña es la mayor fuente de fibra para la industria de pulpa y papel en México. Las pulpas de bagazo se emplean en la mayoría de los grados de papel: escritura, toallas e higiénico, recubierto y muchos otros. El almacenamiento y manejo de las fibras de bagazo son factores críticos en el rendimiento, calidad y propiedades de las pulpas de bagazo, debido a que éste es el residuo resultante de los molinos del ingenio y es un material estacional, por lo tanto, su almacenamiento durante la zafra azucarera es fundamental para las operaciones en una planta de pulpa y papel. Sin embargo, las fibras cortas de bagazo sufren degradación por microorganismos y procesos fermentativos durante el periodo de almacenamiento. El objetivo del presente trabajo es evaluar el impacto del almacenamiento de 12 meses en las propiedades mecánicas de la pulpa de bagazo resultante comparadas con aquella blanqueada y sin blanquear obtenidas de bagazo fresco. El pulpeo a la sosa aplicado al bagazo almacenado obtuvo los mejores resultados en cuanto a rendimiento, grado de deslignificación y propiedades ópticas; sin embargo, para pulpa de bagazo fresco las propiedades mecánicas y de drenado se incrementaron considerablemente (índice de explosión $42 \%$, resistencia al doblez $610 \%$, longitud de ruptura 53\%, Freeness $10 \%$, tiempo de drenado $4.2 \%$, porosidad $20 \%$ y grado de refinación $17.6 \%$ ) sin embargo, el rasgado disminuyó $14.2 \%$. El análisis mostró la influencia del método actual de almacenamiento y manejo sobre las propiedades de la pulpa al disminuir las resistencias y la necesidad de sistematizar y calendarizar el manejo de fibra en la planta para producir pulpas químicas de bagazo de calidad, para blanqueo convencional o ECF y TCF.
\end{abstract}

\section{Descriptores}

- pulpas de bagazo

- almacenamiento

- blanqueo ECF

- propiedades mecánicas

- evaluación de pulpas 


\begin{abstract}
Sugarcane bagasse is now a major source of fibre for pulp and papermaking in Mexico, bagasse pulps are used for all grades of paper: writing, toilet tissue, towelling, glassine, and others. The storage and handling of fibres are critical factors in the resulting pulp yield and quality because bagasse is a fibrous residue that remains after crushing the stalks, and contains short fibres and is a seasonal raw material. The storage produced at harvest time becomes necessary when it is used for operations that are carried out continuously throughout the year, such as pulp and paper production. Nevertheless the sugar cane bagasse fibers suffer severe degradation while in storage due to the action of undesirable micro-organisms. The causes for the deterioration as fermentation proceed and the methods of storing and handling the bagasse to minimize this degradation are discussed. The purpose of this paper is to analyze the impact of quality from bagasse stored for 12 months on mechanical pulp properties compared with those of corresponding bleached and unbleached pulp made from mills bagasse (fresh bagasse). Soda pulping from storaged bagasse gave the best results in yield, degree of delignification and brightness; but it was found that all of the pulp strength and drainage properties were increased to the fresh bagasse pulp (burst index 42\%, folding endurance $610 \%$ and breaking length 53\%, Freeness $10 \%$, drainage time of pulp $4.2 \%$, porosity $20 \%$ and refinement degree $17.6 \%$ ) however, tear index was reduced $14.2 \%$. The analysis showed the influence of storage method on the mechanical properties of bleached and unbleached bagasse pulp and the need for an improved wet bulk storage system to produce acceptable quality chemical bagasse pulps from conventional bleaching method (CEH sequence) and ECF y TCF pulps.
\end{abstract}

\section{Introducción}

El bagazo es el residuo del proceso de fabricación del azúcar a partir de la caña, el remanente de los tallos de la caña después de ser extraído el jugo azucarado que ésta contiene; se ha empleado tradicionalmente en los países azucareros como materia prima para la producción de energía en las calderas de los ingenios o centrales azucareros y su empleo en la manufactura de papel inició hace más de 150 años además de la fabricación de paneles aglomerados de fibras y de partículas y celulosa para derivados farmacéuticos y aditivos de alimentos (Rutiaga et al., 2002 y Jiménez, 1997).

Las fibras son el constituyente fundamental del papel y tienen influencia en la resistencia de este producto. Para tener un buen papel tanto desde el punto de vista de su resistencia como de calidad de impresión, es importante hacer una buena combinación de fibra larga y corta. Por esto, las materias primas fibrosas como el bagazo deben de reunir determinados requisitos de índole técnica y económica como composición química, reactividad frente a los agentes de pulpeo y propiedades anatómico-morfológicas. Ya que a pesar de sus características de fibra corta, es posible obtener pulpa blanqueada y papeles para impresión y escritura de alta calidad a partir del bagazo en combinación con fi- bras largas vírgenes y recicladas, como lo demuestra la existencia de plantas basadas en esta materia prima en el mundo que operan y compiten exitosamente en el mercado de productos de celulosa, papel y cartón (Rainey et al., 2004; Molina, 2002; Sharma et al., 2000 y Hunter, 2001).

Triana (1990) mencionó que el bagazo está constituido por cuatro fracciones: Fibra o bagazo (45 \%), sólidos no solubles (2-3\%), sólidos solubles (2-3\%) y agua (49-51\%). La parte designada por fibra está compuesta de toda la fracción sólida orgánica, insoluble en agua, portadora de los elementos estructurales necesarios para la industria de pulpa y papel. Esta fracción está influenciada por las condiciones de procesamiento agrícola de la caña, tipo de corte y recolección, manejo del bagazo y almacenamiento. La fracción denominada fibra por el azucarero, está constituida desde el punto de vista morfológico por la fracción fibra verdadera y por meollo (médula o parénquima). El desmedulado constituye un paso imprescindible al mejorar el bagazo para la producción de pulpas, aumenta el rendimiento y mejora la blancura y las propiedades de resistencia del papel resultante.

El contenido de médula en el bagazo tiene incidencia negativa sobre el almacenamiento de la materia prima para el periodo de no zafra. Mientras mayor sea la 
remoción de la médula, menor o más controlada será la fermentación, a causa de una mayor eliminación de azúcares residuales e impurezas y por lo tanto, es importante asegurar las propiedades del bagazo para su conversión en pulpa y papel (Zegarra, 1977; Schmidt, 1977 y Atchison, 1962).

\section{Conservación y almacenamiento del bagazo de caña}

En la mayor parte de las zonas azucareras del mundo el procesamiento y corte de la caña de azúcar es una actividad temporal. El almacenamiento del bagazo producido en la época de zafra se hace necesario cuando éste se va a utilizar para operaciones continuas durante un año en la industria de pulpa y papel. Desde el punto de vista conceptual, almacenamiento consiste en guardar el bagazo durante un espacio de tiempo para ser empleado en un momento determinado, sin sufrir transformaciones en cuanto a calidad, que impidan su uso posterior, todo esto debe lograrse con el menor costo posible entre los períodos de molienda de la caña de azúcar.

En la literatura especializada se reportan ampliamente los principales métodos de almacenamiento de bagazo en forma seca y húmeda (Celotex, Taiwán, Cuba 9, Begatex, Ritter, Cusi entre otros) (Rainey et al., 2006), (Atchison et al., 2004, 1971, 1962), (Kadam, 2000), (Rangan et al. 1998, 1989) y Lois (1995) y las ventajas y desventajas de cada uno de ellos, así como los efectos sobre las propiedades físicas de las pulpas resultantes, los efectos inevitables del deterioro del bagazo en el almacenamiento en la forma de oscurecimiento de las fibras por acción de los hongos y la degradación de la misma que provoca la prehidrólisis ácida de los materiales celulósicos. El almacenaje húmedo o mojado de la masa formando pilas es el de uso generalizado (Yadav et al., 2003; Sharma et al., 2000; Lois, 1985; Cusi, 1980; Schmidt, 1977 y Salaber et al., 1971) y se emplea en la mayoría de las plantas con el fin de reducir los costos de manejo y almacenamiento.

El bagazo en estas condiciones de humedad (48 $52 \%$ ) constituye un rico sustrato para el desarrollo de microorganismos y tienen lugar varias reacciones simultáneas, debido a los factores ambientales dando lugar a la generación de calor (tabla 1). En comparación con la madera, el deterioro del bagazo tiene lugar de una manera diferente, debido a su contenido inicial en humedad, sacarosa, azúcares invertidos, proteínas residuales, minerales y a la heterogeneidad de sus tejidos, que se agrava por el daño sufrido durante la operación de la caña. Estas condiciones favorecen procesos de oxi- dación química, disminuye el grado de brillantez y pérdidas en peso del bagazo almacenado, con valores que alcanzan hasta 20 - $30 \%$ en condiciones pobres de almacenamiento y al mismo tiempo, ocurren grandes afectaciones de la calidad de la fibra (Sharma et al., 2000)

Tabla 1. Reacciones químicas y producción de calor (Rangan, 1989, Cusi, 1980, Schmidt, 1977)

\begin{tabular}{cc}
$\begin{array}{c}\text { Reacciones en } \\
\text { una pila } \\
\text { de bagazo almacenado }\end{array}$ & $\begin{array}{c}\text { Calor generado } \\
(\mathrm{Cal} / \text { gr Mol de } \\
\text { Sacarosa })\end{array}$ \\
\hline $\mathrm{C}_{12} \mathrm{H}_{22} \mathrm{O}_{11}+12 \mathrm{O}_{2} \rightarrow 12\left(\mathrm{CO}_{2}\right)+11\left(\mathrm{H}_{2} \mathrm{O}\right)$ & 1348.2 \\
Oxidación de la sacarosa & 8.32 \\
$\mathrm{C}_{12} \mathrm{H}_{22} \mathrm{O}_{11}+\mathrm{H}_{2} \mathrm{O} \rightarrow 2\left(\mathrm{C}_{6} \mathrm{H}_{12} \mathrm{O}_{6}\right)$ & \\
Sacarosa $\rightarrow$ glucosa + fructosa & 1339.88 \\
$2\left(\mathrm{C}_{6} \mathrm{H}_{12} \mathrm{O}_{6}\right)+6 \mathrm{O}_{2} \rightarrow 6\left(\mathrm{CO}_{2}\right)+6\left(\mathrm{H}_{2} \mathrm{O}\right)$ & \\
Oxidación de la glucosa + fructosa & 33.16 \\
$2\left(\mathrm{C}_{6} \mathrm{H}_{12} \mathrm{O}_{6}\right) \rightarrow 4\left(\mathrm{C}_{2} \mathrm{H}_{5} \mathrm{OH}\right)+4\left(\mathrm{CO}_{2}\right)$ & \\
$\mathrm{Glucosa}+$ fructosa $\rightarrow$ etanol & 32.68 \\
$2\left(\mathrm{C}_{6} \mathrm{H}_{12} \mathrm{O}_{6}\right) \rightarrow 4\left(\mathrm{CH}_{3} \mathrm{CH}(\mathrm{OH}) \mathrm{COOH}\right)$ & \\
$\mathrm{Glucosa}+$ fructosa $\rightarrow$ acido láctico & \\
$4\left(\mathrm{C}_{2} \mathrm{H}_{5} \mathrm{OH}\right)+4 \mathrm{O} 2 \rightarrow 4 \mathrm{CH} \mathrm{HOOH}_{3} \mathrm{CO}+4 \mathrm{H}_{2} \mathrm{O}$ & 470.64 \\
Etanol $\rightarrow$ acido acético & \\
\hline
\end{tabular}

Sin embargo, aunque existen trabajos científicos complementarios a los básicos (Cusi, 1980; Liese, 1978; Hesch, 1976 y Atchison, 1971) reportados por numerosos autores sobre la cinética y los efectos totales de degradación del bagazo de caña almacenado (Macaskill 2001; Hunter 2001 y Chen, 2009) y su calidad intrínseca para la industria de celulosa y papel (Atchison, 2004); en México no existen estudios que permitan establecer claramente la relación entre los objetivos de almacenamiento de bagazo, deterioro, deslignificación, blancura, resistencias mecánicas, costos y calidad del producto final; por lo que el objetivo del presente trabajo fue evaluar el efecto del almacenamiento de bagazo de caña de azúcar sobre las propiedades físicas de la pulpa celulósica resultante blanqueada y sin blanquear.

\section{Materiales y métodos}

Se empleó pulpa de bagazo de caña de azúcar procedente de una planta papelera de Veracruz, México, blanqueada y sin blanquear, obtenida bajo condiciones de manejo industrial. Es decir, bagazo desmedulado almacenado durante el periodo de no zafra, pulpeo a la sosa y blanqueo convencional a base de compuestos clorados mediante la secuencia CEH (cloración en medio acido, extracción alcalina y blanqueo con hipoclorito alcalino). 
También se obtuvieron pulpas de bagazo de caña blanqueada y sin blanquear a partir de bagazo fresco procedente del periodo de la zafra del ingenio azucarero José María Martínez S.A. de Tala, Jalisco, México desmedulado con la relación Fibra útil/ Médula 87/13, el cual fue sometido a un método de pulpeo o deslignificacion por el proceso a la sosa $(\mathrm{NaOH})$ simulando las condiciones industriales con las variables: $13 \%$ de reactivos como $\mathrm{NaOH}$ sobre la base del peso seco de bagazo de caña (100 grs b.s), Hidromódulo o relación de baño 7:1, Temperatura máxima $170{ }^{\circ} \mathrm{C}$ y tiempo a temperatura máxima 30 minutos.

Transcurrido el tiempo de cocción, se recuperó el licor negro para su posterior análisis mediante la técnica TAPPI T 625 om-85: "análisis del licor negro de los procesos a la sosa y al sulfato". Las pulpas se lavaron para eliminar el licor negro, posteriormente se desintegraron en un pulper y se transfirieron a un depurador de diafragma con abertura de ranuras de 0.15 milésimas de pulgada propio para fibras de plantas anuales. Posteriormente fueron homogeneizadas en un equipo Hobbart y se toma una muestra para determinar humedad por pérdida de peso, rendimiento y el grado de deslignificación (número de Kappa), de acuerdo con la técnica TAPPI T $236 \mathrm{~cm}-85$.

La pulpa morena o cruda de bagazo resultante se blanqueó mediante la secuencia ECF, libre de cloro elemental, ODP (deslignificación con oxígeno - blanqueo con dióxido de cloro - blanqueo con peróxido), debido a que las fábricas de pulpa blanqueada, tanto las existentes como las de nueva creación se dirigen hacia el empleo de agentes de blanqueo no agresivos en las operaciones de blanqueo en sustitución del cloro y sus derivados, los cuales producen efluentes altamente tóxicos y de elevada carga orgánica difíciles de tratar por vía externa e incluso de recircular dentro del propio sistema (Mariani, 2005). Posterior al blanqueo, la pulpa recibió un tratamiento similar a la pulpa morena.

\section{Evaluación de pulpas de bagazo de caña}

Sanjuán (1997) mencionó que para caracterizar a las pulpas de bagazo de caña con respecto a su calidad, capacidad de procesamiento y adaptabilidad para varios usos es necesario evaluar propiedades de resistencia a la tensión, explosión, rasgado, doblez, de superficie, como lisura; ópticas como blancura, opacidad, brillo y color, de permeabilidad a fluidos como agua, aceite y gases como aire y drenabilidad.

La mayor parte de los métodos de prueba aplicados a los productos de pulpa y papel están estandarizados; en el cuadro 2 se muestran las técnicas de prueba que se utilizaron en el presente estudio. Estos corresponden a los métodos y técnicas analíticas de la Asociación Americana de Técnicos de las Industrias de Pulpa y Papel (TAPPI), para determinar y correlacionar las propiedades de la pulpa de bagazo de caña bajo manejo y almacenamiento y fresco, en todos los casos de pruebas el peso base promedio de las hojas de pulpa fue de $60 \mathrm{~g} / \mathrm{m}^{2}$ y el tamaño de muestra para cada determinación se estableció de acuerdo a la Técnica TAPPI T 220 sp-96 (Pruebas físicas de hojas de pulpa).

Tabla 2. Técnicas TAPPI utilizadas en la determinación de propiedades físicas de la pulpa de bagazo (Sanjuan, 1997)

\begin{tabular}{lc}
\multicolumn{1}{c}{ Análisis } & Técnica \\
\hline No. Kappa en pulpa & T 236 cm - 85. \\
Lignina en pulpa & T 222 om - 88 \\
Longitud de fibras de pulpa por clasificación & T-233 cm - 82 \\
Formación de hojas para pruebas físicas & T-205 om - 88 \\
Acondicionamiento de las hojas & T -402 om - 93 \\
Índice de explosión & T-403 om - 91 \\
Resistencia al rasgado & T-414 om - 88 \\
Blancura ISO & T-218 om - 91 \\
Opacidad & T-519 om - 96 \\
Viscosidad CED & T-230 om - 89 \\
Reversión de blancura ISO & T-um - 200 \\
Resistencia al doblez & T - 423 \\
Refinación de pulpas celulósicas & T-248-wd-97 \\
Grado de refinación ( ${ }^{\circ}$ SR) & SCAN-m3:65 \\
Capacidad de drenado (Freeness) (mL C.S.F) & T-227 \\
Tiempo de drenado en pulpas & T-205-sp-95 \\
Porosidad de pulpas & T-460-om-96 \\
\hline
\end{tabular}

\section{Refinación de pulpas celulósicas}

La refinación o batido, fue el trabajo mecánico que se aplicó a las fibras para separarlas, fibrilarlas, cortarlas e hidratarlas para mejorar las propiedades de resistencia y formación. En todos los equipos de refinación existen los mismos elementos de trabajo: barras separadas que cubren un elemento en movimiento y un elemento estacionario, entre los cuales se hace pasar la fibra, aprisionándola y ejerciendo una fuerza mecánica sobre ella (Sanjuán, 1997). El efecto de la acción mecánica, incrementa la velocidad de aceptación del agua, con lo cual, se logran más superficies de contacto, posteriormente se produce el acortamiento y la destrucción de las fibras. El control y evaluación durante el proceso de refinación, y al término de éste, se puede realizar determinando el grado, capacidad de drenado o desgote de las pulpas por efecto del tratamiento mecánico de refinación. La medición de éste se realizó mediante el método Shopper Riegler ( ${ }^{\circ}$ S.R.) y el de Canadian Standard Freeness (mL C.S.F.) debido a que estos parámetros permiten: 
1) determinar la longitud promedio de la pulpas, a mayor cantidad de fibra larga mayor Freeness y menor ${ }^{\circ}$ S.R.

2) el daño a las fibras como consecuencia del tratamiento de la materia prima, pulpeo, blanqueo o secado y

3) la energía de refinación requerida para alcanzar ciertas propiedades de drenado en las operaciones de lavado de pulpa, la preparación de pasta y la formación en la maquina de papel (runnability) (Savcor, 2007). El tratamiento se llevó a cabo en un molino Jokro con 16 g de pulpa B.S. llevados a 265 gr. con agua en un contenedor liso en el que se encuentra un rodillo acanalado con excentricidad que gira a determinado tiempo (minutos).

\section{Resultados y discusión}

Se entiende que el diseño de un sistema eficiente en las condiciones de almacenamiento del bagazo es un paso fundamental para la preservación de la fibra y para el control de las pérdidas, por lo que el almacenaje del bagazo debe ser concebido como una etapa más del proceso productivo (Yadav et al., 2003). Las pulpas evaluadas (tabla 3) difieren entre sí, ya que la pulpa industrial presenta de inicio, menores resistencias mecánicas, a excepción del rasgado y propiedades de drenado inferiores; sin embargo, la pulpa requerirá menor carga de reactivos de blanqueo al presentar mayor blancura y menor contenido de lignina después de la etapa de cocción, aunque menor viscosidad intrínseca como indicativo de su grado de degradación, debido a los cambios que la fibra presenta durante el almacenamiento y su posterior caída en las resistencias mecáni-

Tabla 3. Propiedades físicas de pulpas de bagazo

\begin{tabular}{lccc}
\hline \multicolumn{1}{c}{ Propiedad / pulpa } & $\begin{array}{c}\text { Bagazo } \\
\text { s/almacenar }\end{array}$ & $\begin{array}{c}\text { Bagazo } \\
\text { industrial }\end{array}$ & $\begin{array}{c}\text { Diferencia } \\
\text { (fresco/almacenado) }\end{array}$ \\
\hline Rendimiento en pulpeo (\%) & 54.5 & 53.0 & $+2.8 \%$ \\
Rechazos (\%) & 4.2 & 4.0 & $+5 \%$ \\
No. Kappa & 18.1 & 11,8 & $+53.4 \%$ \\
Lignina Residual (\%) & 2.72 & 1.77 & $+53.7 \%$ \\
Viscosidad CED & 36.8 & 21.4 & $+72 \%$ \\
Grado de refinación ( ${ }^{\circ}$ S.R.) & 14 & 17 & $-17.6 \%$ \\
Freeness (mL C.S.F.) & 721 & 653 & $+10 \%$ \\
Largo de ruptura ( Metros) & 5617 & 3672 & $+53 \%$ \\
Índice de explosión (kPa.m $\left.{ }^{2} / \mathrm{g}\right)$ & 3.59 & 2.53 & $+42 \%$ \\
Índice de rasgado (mN.m $\left.{ }^{2} / \mathrm{g}\right)$ & 7.85 & 9.15 & $-14.2 \%$ \\
Resistencia al doblez (No.) & 213 & 30 & $+610 \%$ \\
Porosidad (S / mL) & 6.0 & 5.0 & $+20 \%$ \\
Tiempo de drenado ( Seg) & 4.55 & 4.75 & $-4.2 \%$ \\
Blancura (\% ISO) & 48.2 & 50.3 & $-4.1 \%$ \\
\hline
\end{tabular}

cas como fue reportado por (Savcor, 2007; Atchinson, 2004; Rangan et al., 1998; García, 1989; Cusi, 1980 y Salaber et al., 1971).

\section{Clasificación de fibras de pulpas}

La longitud de las fibras es una propiedad que influye significativamente en su potencial para fabricar productos de pulpa y papel, ya que una mayor fracción de fibra larga le confiere propiedades de resistencia mecánica a las pulpas celulósicas y al papel resultante. Se determinó que la pulpa de bagazo almacenado presentó un signicativo contenido menor de la fracción de fibras largas como consecuencia de la degradación del bagazo durante el periodo de almacenamiento (tabla 4) lo cual se compensa directamente en la etapa de refinación al requerir menor tratamiento mecánico y energía para alcanzar un determinado grado de refinación ('S.R.) y (Freeness) (tabla 5).

\section{Refinación de pulpas individuales}

La pulpa industrial alcanza valores de refinación más altos (menor Freeness y mayor ${ }^{\circ}$ S.R.) en un menor tiempo con mayor generación de finos, debido al rompimiento de células y a la baja cantidad de fibras largas que la pulpa de bagazo obtenida en el laboratorio; en esta última, el tratamiento tendió hacia la flexibilidad, el hinchamiento y la fibrilación y no el corte y por el contrario la pulpa industrial mostró rigidez, por lo tanto bajas resistencias mecánicas a un mismo grado de refinación (Aguilar, 2002). En la literatura se recomienda no refinar a más de $45^{\circ} \mathrm{SR}$ (255 mL C.S.F.), ya que se reduce el rasgado significativamente expresado como índice; entonces la refinación a valores muy altos de trabajo mecánico sobre las fibras de bagazo puede conllevar a la disminución de la longitud promedio por el acortamiento de las fibras, lo que se reflejó en las propiedades mecánicas (Sharkawy et al., 2005; Yadav et al., 2003 y Zegarra, 1977) (tablas 6 a 11), ayudando así a que la mayoría de las propiedades se incrementen y a diferencia de la pulpa procedente del sistema de almacenamiento la medición de la refinación y el Freeness manifiestan que han sido cortadas como consecuencia del proceso de refinación y tienen por lo tanto, poca resistencia; sin embargo, el tiempo de 
Tabla 4. Clasificación de fibras de pulpas individuales (\% Retenido)

\begin{tabular}{cccc}
\hline Malla & $\begin{array}{c}\text { Bagazo } \\
\text { s/almacenar }\end{array}$ & $\begin{array}{c}\text { Bagazo } \\
\text { industrial }\end{array}$ & $\begin{array}{c}\text { Diferencia } \\
\text { (fresco/ } \\
\text { almacenado) }\end{array}$ \\
\hline 30 & 18.9 & 0.5 & $+3680 \%$ \\
50 & 34.6 & 26.9 & $+28.6 \%$ \\
100 & 26.1 & 30.6 & $-14.7 \%$ \\
200 & 7.9 & 19.7 & $-60 \%$ \\
$<200$ & 12.5 & 22.4 & $-44.2 \%$ \\
\hline
\end{tabular}

Tabla 5. Grado de refinación de pulpas de bagazo

\begin{tabular}{ccccc}
\hline $\begin{array}{c}\text { Tiempo de } \\
\text { refinación, } \\
\text { (minutos) }\end{array}$ & \multicolumn{2}{c}{$\begin{array}{c}\text { Bagazo } \\
\text { industrial }\end{array}$} & \multicolumn{2}{c}{$\begin{array}{c}\text { Bagazo } \\
\text { s/almacenar }\end{array}$} \\
\hline 0 & ${ }^{\circ}$ S.R. & mL C.S.F. & ${ }^{\circ}$ S.R. & mL C.S.F. \\
3 & 17 & 653 & 14 & 721 \\
6 & 24 & 520 & 20 & 592 \\
12 & 31 & 415 & 23 & 537 \\
18 & 50 & 211 & 32 & 401 \\
& 66 & 98 & 48 & 229 \\
\hline
\end{tabular}

drenado y la porosidad permanecen con valores superiores a la pulpa obtenida de bagazo fresco, esto puede deberse a lo reportado por Atchison et al., (2004), Sharma et al. (2000) y Rangan (1989), quienes concluyeron que durante el almacenamiento de bagazo, las sustancias solubles del bagazo disminuyen y la proporción de holocelulosa se incrementa, por otro lado, los enlaces de la lignina se debilitan favoreciendo la deslignificación y el blanqueo (tabla 3); sin embargo, las menores resistencias mecánicas son debidas a que la degradación durante el almacenamiento toma lugar directamente en la celulosa y hemicelulosas mediante la hidrólisis, lo cual se manifiesta en una menor viscosidad intrínseca (Savcor 2007) y (Salaber et al., 1971)

El comportamiento anterior se generó de la misma forma para pulpas blanqueadas; sin embargo, la secuencia de blanqueo aplicada es fundamental, ya que según Mariani (2005) en el mercado internacional de pulpas blanqueadas se hace importante concebir nuevas secuencias de blanqueo libres de cloro para todo tipo de pulpa química blanqueada, por lo que aunque la pulpa sin almacenar la secuencia ODP llevó a una parcial degradación de la pulpa de bagazo a diferencia de la secuencia $\mathrm{CEH}$, aplicada en la pulpa de bagazo almacenado (tabla 12). La pulpa ODP presentó propiedades ópticas superiores a un menor impacto por efecto de los efluentes altamente contaminantes por la alta cantidad de organo-clorados y cloroformo (AOX) que se generan en las etapas, además del fuerte impacto de color que genera la etapa de extracción alcalina (tabla 13) y en la pulpa resultante, un mayor contenido de fi- bra larga (tabla 14) lo que se manifestó en una menor energía en la refinación para alcanzar el mismo grado de Freeness y en el incremento en la propiedades de resistencia mecánica en relación a la pulpa CEH (tablas 15 a 21).

Tabla 6. Índice de explosión de pulpas de bagazo de caña $\left(\mathrm{KPa} \mathrm{m}{ }^{2} / \mathrm{g}\right)$

\begin{tabular}{cccc}
\hline $\begin{array}{c}\text { Grado de } \\
\text { refinación ( }{ }^{\circ} \text { S.R.) }\end{array}$ & $\begin{array}{c}\text { Freeness } \\
(\mathrm{mL} \text { C.S.F. })\end{array}$ & $\begin{array}{c}\text { Bagazo } \\
\text { industrial }\end{array}$ & $\begin{array}{c}\text { Bagazo } \\
\text { s/almacenar }\end{array}$ \\
\hline 17 & 653 & 2.53 & 4.58 \\
23 & 537 & 3.47 & 6.55 \\
32 & 401 & 4.72 & 7.22 \\
37 & 339 & 4.94 & 7.32 \\
48 & 229 & 5.43 & 7.53 \\
\hline
\end{tabular}

Tabla 7. Longitud de ruptura de pulpas individuales (metros)

\begin{tabular}{cccc}
\hline $\begin{array}{c}\text { Grado de } \\
\text { refinación ('S.R.) }\end{array}$ & $\begin{array}{c}\text { Freeness } \\
(\text { mL C.S.F. })\end{array}$ & $\begin{array}{c}\text { Bagazo } \\
\text { industrial }\end{array}$ & $\begin{array}{c}\text { Bagazo s/ } \\
\text { almacenar }\end{array}$ \\
\hline 17 & 653 & 3672 & 6690 \\
23 & 537 & 4243 & 8838 \\
32 & 401 & 6643 & 9146 \\
37 & 339 & 6546 & 9112 \\
48 & 229 & 6324 & 9039 \\
\hline
\end{tabular}

Tabla 8. Índice de rasgado de pulpas individuales $\left(\mathrm{mN} \mathrm{m}{ }^{2} / \mathrm{g}\right)$

\begin{tabular}{cccc}
\hline $\begin{array}{c}\text { Grado de } \\
\left.\text { refinación ( }{ }^{\circ} \text { S.R. }\right)\end{array}$ & $\begin{array}{c}\text { Freeness } \\
(\text { mL C.S.F.) }\end{array}$ & $\begin{array}{c}\text { Bagazo } \\
\text { industrial }\end{array}$ & $\begin{array}{c}\text { Bagazo s/ } \\
\text { almacenar }\end{array}$ \\
\hline 17 & 653 & 9.15 & 9.35 \\
23 & 537 & 8.87 & 12.35 \\
32 & 401 & 8.51 & 9.78 \\
37 & 339 & 8.31 & 9.38 \\
48 & 229 & 7.85 & 8.50 \\
\hline
\end{tabular}

Tabla 9. Resistencia al doblez de pulpas individuales (núm. dobles pliegues)

\begin{tabular}{cccc}
\hline $\begin{array}{c}\text { Grado de } \\
\text { refinación ( }{ }^{\circ} \text { S.R.) }\end{array}$ & $\begin{array}{c}\text { Grado de } \\
\text { refinación (C.S.F.) }\end{array}$ & $\begin{array}{c}\text { Bagazo } \\
\text { industrial }\end{array}$ & $\begin{array}{c}\text { Bagazo s/ } \\
\text { almacenar }\end{array}$ \\
\hline 17 & 653 & 30 & 288 \\
23 & 537 & 84 & 437 \\
32 & 401 & 156 & 689 \\
37 & 339 & 172 & 721 \\
48 & 229 & 206 & 792 \\
\hline
\end{tabular}

Tabla 10. Tiempo de drenado de pulpas individuales (s)

\begin{tabular}{cccc}
\hline $\begin{array}{c}\text { Grado de } \\
\text { refinación }\left({ }^{\circ} \text { S.R. }\right)\end{array}$ & $\begin{array}{c}\text { Freeness } \\
(\text { mL C.S.F.) }\end{array}$ & $\begin{array}{c}\text { Bagazo } \\
\text { industrial }\end{array}$ & $\begin{array}{c}\text { Bagazo s/ } \\
\text { almacenar }\end{array}$ \\
\hline 17 & 653 & 4.75 & 5.14 \\
23 & 537 & 5.93 & 6.32 \\
32 & 401 & 7.70 & 8.05 \\
37 & 339 & 9.07 & 8.87 \\
48 & 229 & 12.08 & 10.66 \\
\hline
\end{tabular}


Tabla 11. Porosidad de pulpas individuales $(\mathrm{s} / 100 \mathrm{~mL})$

\begin{tabular}{|c|c|c|c|}
\hline $\begin{array}{c}\text { Grado de } \\
\text { refinación ( }{ }^{\circ} \text { S.R.) }\end{array}$ & $\begin{array}{l}\text { Freeness } \\
\text { (mL C.S.F.) }\end{array}$ & $\begin{array}{c}\text { Bagazo } \\
\text { industrial }\end{array}$ & $\begin{array}{l}\text { Bagazo s/ } \\
\text { almacenar }\end{array}$ \\
\hline 17 & 653 & 5 & 21 \\
\hline 23 & 537 & 26 & 53 \\
\hline 32 & 401 & 53 & 138 \\
\hline 37 & 339 & 79 & 223 \\
\hline 48 & 229 & 135 & 410 \\
\hline
\end{tabular}

\section{Propiedades de Pulpas blanqueadas}

Tabla 12. Propiedades físicas de pulpas blanqueadas de bagazo

\begin{tabular}{|c|c|c|c|}
\hline $\begin{array}{l}\text { Propiedad / } \\
\text { pulpa }\end{array}$ & $\begin{array}{l}\text { Bagazo s/ } \\
\text { almacenar }\end{array}$ & $\begin{array}{c}\text { Bagazo } \\
\text { industrial } \\
\end{array}$ & $\begin{array}{c}\text { Diferencia } \\
\text { (fresco/ } \\
\text { almacenado) }\end{array}$ \\
\hline Viscosidad CED & 11.2 & 17.0 & $-34 \%$ \\
\hline $\begin{array}{l}\text { Grado de refinación } \\
\text { ('S.R.) }\end{array}$ & 20 & 21 & $-4.8 \%$ \\
\hline $\begin{array}{l}\text { Freeness } \\
(\mathrm{mL} \text { C.S.F.) }\end{array}$ & 592 & 574 & $-3.1 \%$ \\
\hline $\begin{array}{l}\text { Largo de ruptura } \\
\text { (Metros) }\end{array}$ & 5070 & 4030 & $+25.8 \%$ \\
\hline $\begin{array}{l}\text { Î́ndice de explosión } \\
\left(\mathrm{kPa} \cdot \mathrm{m}^{2} / \mathrm{g}\right)\end{array}$ & 4.17 & 2.75 & $+51.6 \%$ \\
\hline $\begin{array}{l}\text { Índice de rasgado } \\
\left(\mathrm{mN} \cdot \mathrm{m}^{2} / \mathrm{g}\right)\end{array}$ & 8.50 & 7.52 & $+13 \%$ \\
\hline $\begin{array}{l}\text { Resistencia al doblez } \\
\text { (Núm.) }\end{array}$ & 127 & 34 & $+273 \%$ \\
\hline $\begin{array}{l}\text { Tiempo de drenado } \\
\text { (s) }\end{array}$ & 5.18 & 5.09 & $+1.8 \%$ \\
\hline $\begin{array}{l}\text { Porosidad } \\
\text { (s/mL) }\end{array}$ & 11.26 & 11.07 & $+1.7 \%$ \\
\hline
\end{tabular}

Tabla 13. Propiedades ópticas de pulpas (\%)

\begin{tabular}{ccc}
\hline & & Bagazo \\
Propiedad & Bagazo industrial & s/almacenar \\
\hline Blancura & 81.2 & 89.9 \\
Opacidad & 76 & 78 \\
Reversión & 77.0 & 88.3 \\
\hline
\end{tabular}

Tabla 14. Clasificación de fibras de pulpas blanqueadas (\%)

\begin{tabular}{cccc}
\hline Malla & $\begin{array}{c}\text { Bagazo } \\
\text { s/almacenar }\end{array}$ & $\begin{array}{c}\text { Bagazo } \\
\text { industrial }\end{array}$ & $\begin{array}{c}\text { Diferencia } \\
\text { (fresco/almacenado) }\end{array}$ \\
\hline 30 & 4.7 & 1.8 & $+161 \%$ \\
50 & 23.3 & 28.3 & -17.6 \\
100 & 39.7 & 36.2 & $+9.7 \%$ \\
200 & 14.7 & 14.6 & $+0.7 \%$ \\
$<200$ & 17.6 & 19.1 & $-7.9 \%$ \\
\hline
\end{tabular}

Tabla 15. Grado de refinación ( ${ }^{\circ}$ S.R. y mL C.S.F.) de pulpas blanqueadas

\begin{tabular}{ccccc}
\hline $\begin{array}{l}\text { Tiempo de } \\
\text { refinación } \\
\text { (Minutos) }\end{array}$ & \multicolumn{2}{c}{$\begin{array}{c}\text { Bagazo industrial } \\
\text { blanqueada }\end{array}$} & \multicolumn{2}{c}{$\begin{array}{c}\text { Bagazo s/almacenar } \\
\text { blanqueada }\end{array}$} \\
\hline 0 & 21 & 574 & 20 & 592 \\
1 & 25 & 504 & 23 & 537 \\
2 & 33 & 388 & 25 & 504 \\
5 & 43 & 275 & 34 & 375 \\
\hline
\end{tabular}

Tabla 16. Longitud de ruptura de pulpas blanqueadas (Metros)

\begin{tabular}{cccc}
\hline $\begin{array}{c}\text { Grado de } \\
\text { refinación ( }\end{array}$ & $\begin{array}{c}\text { Freeness } \\
\text { (mL C.S.F.) }\end{array}$ & $\begin{array}{c}\text { Bagazo } \\
\text { industrial }\end{array}$ & $\begin{array}{c}\text { Bagazo s/ } \\
\text { almacenar }\end{array}$ \\
\hline 21 & 574 & 4030 & 5070 \\
25 & 504 & 5070 & 6727 \\
29 & 442 & 6109 & 7140 \\
33 & 388 & 7149 & 7656 \\
\hline
\end{tabular}

Tabla 17. Índice de explosión de pulpas blanqueadas (KpaM²/g)

\begin{tabular}{cccc}
\hline $\begin{array}{c}\text { Grado de } \\
\text { refinación }\left({ }^{\circ} \text { S.R. }\right)\end{array}$ & $\begin{array}{c}\text { Freeness } \\
(\text { mL C.S.F. })\end{array}$ & $\begin{array}{c}\text { Bagazo } \\
\text { industrial }\end{array}$ & $\begin{array}{c}\text { Bagazo } \\
\text { s/almacenar }\end{array}$ \\
\hline 21 & 574 & 2.75 & 4.17 \\
25 & 504 & 3.41 & 5.72 \\
29 & 442 & 4.07 & 6.09 \\
33 & 388 & 4.73 & 6.56 \\
\hline
\end{tabular}

Tabla 18. Índice de rasgado de pulpas blanqueadas ( $\mathrm{mN} \mathrm{m}$ ²/g)

\begin{tabular}{cccc}
\hline $\begin{array}{c}\text { Grado de } \\
\text { refinación (' }\end{array}$ & $\begin{array}{c}\text { Freeness } \\
(\mathrm{mL} \text { C.S.F.) }\end{array}$ & $\begin{array}{c}\text { Bagazo } \\
\text { industrial }\end{array}$ & $\begin{array}{c}\text { Bagazo } \\
\text { s/almacenar }\end{array}$ \\
\hline 21 & 574 & 7.52 & 8.50 \\
25 & 504 & 5.81 & 7.85 \\
29 & 442 & 5.53 & 7.27 \\
33 & 388 & 5.23 & 6.54 \\
\hline
\end{tabular}

Tabla 19. Resistencia al doblez de pulpas blanqueadas (dobles pliegues)

\begin{tabular}{cccc}
\hline $\begin{array}{c}\text { Grado de } \\
\text { refinación ( }\end{array}$ 'S.R.) & $\begin{array}{c}\text { Freeness } \\
\text { (mL C.S.F.) }\end{array}$ & $\begin{array}{c}\text { Bagazo } \\
\text { industrial }\end{array}$ & $\begin{array}{c}\text { Bagazo } \\
\text { s/almacenar }\end{array}$ \\
\hline 21 & 574 & 34 & 127 \\
25 & 504 & 52 & 321 \\
29 & 442 & 70 & 322 \\
33 & 388 & 88 & 367 \\
\hline
\end{tabular}

Tabla 20. Tiempo de drenado de pulpas blanqueadas (s)

\begin{tabular}{cccc}
\hline $\begin{array}{c}\text { Grado de } \\
\text { refinación ( }\end{array}{ }^{\circ}$ S.R.) & $\begin{array}{c}\text { Freeness } \\
\text { (mL C.S.F.) }\end{array}$ & $\begin{array}{c}\text { Bagazo } \\
\text { industrial }\end{array}$ & $\begin{array}{c}\text { Bagazo } \\
\text { s/almacenar }\end{array}$ \\
\hline 21 & 574 & 5.09 & 5.21 \\
25 & 504 & 6.11 & 6.86 \\
29 & 442 & 7.19 & 7.53 \\
33 & 388 & 8.27 & 8.19 \\
\hline
\end{tabular}

Tabla 21. Porosidad de pulpas blanqueadas (s/100 mL)

\begin{tabular}{cccc}
\hline $\begin{array}{c}\text { Grado de } \\
\text { refinación ( }\end{array}{ }^{\text {S }}$.R.) & $\begin{array}{c}\text { Freeness } \\
\text { (mL C.S.F.) }\end{array}$ & $\begin{array}{c}\text { Bagazo } \\
\text { industrial }\end{array}$ & $\begin{array}{c}\text { Bagazo } \\
\text { s/almacenar }\end{array}$ \\
\hline 21 & 574 & 11.1 & 11.3 \\
25 & 504 & 63.3 & 57.9 \\
29 & 442 & 149.4 & 106.3 \\
33 & 388 & cerrado & cerrado \\
\hline
\end{tabular}

\section{Conclusiones}

Este trabajo determinó los efectos de los sistemas de almacenamiento de fibras de bagazo en las propiedades físicas de las pulpas. Si bien, la pulpa a la sosa de bagazo almacenado obtuvo los mejores resultados en cuanto a rendimiento y rechazos de cocción, grado de 
deslignificación y propiedades ópticas, debido al efecto parcial de degradación de los enlaces moleculares entre la lignina, hemicelusoas y celulosa, sus propiedades mecánicas y de drenado fueron inferiores con relación a la pulpa de bagazo fresco donde se incrementaron considerablemente sus propiedades para pulpa morena (Índice de explosión 42\%, resistencia al doblez $610 \%$, longitud de ruptura $53 \%$, Freeness $10 \%$, tiempo de drenado $4.2 \%$, porosidad $20 \%$ y grado de refinación $17.6 \%$ ) y blanqueada (Índice de explosión 51\%, resistencia al doblez $273 \%$, longitud de ruptura $25.8 \%$, rasgado $13 \%$, tiempo de drenado $1.8 \%$, porosidad $1.7 \%$ ) a una mayor blancura y opacidad como consecuencia de un mayor contenido de fibras largas. El análisis mostró la influencia del método actual de almacenamiento y manejo sobre las propiedades de la pulpa al disminuir las resistencias y la necesidad de sistematizar y calendarizar el manejo y conservación de las propiedades iniciales del bagazo durante las etapas de zafra y no zafra en la planta para producir pulpas químicas de bagazo de calidad tanto para blanqueo convencional o ECF y TCF para obtener el producto papelero final.

\section{Agradecimientos}

Por la colaboración y apoyo decisivo para la realización de este estudio al personal del Departamento de Madera, Celulosa y Papel Ing. Karl Augustin Grellmann de la Universidad de Guadalajara Jalisco México y en especial al C. Dr. Rubén Sanjuán Dueñas.

\section{Referencias}

Aguilar R.N. Celulosa blanqueada ECF y TCF de cartón kraft reciclado. Ciencia Forestal en México. 27(91):83-96. 2002.

Atchison J.E., Hettenhaus J.E. Innovative Methods for Corn Stover Collecting, Handling, Storing and Transporting [en línea] National Renewable Energy Laboratory, 2004. Disponible en: http://www.osti.gov/bridge

Atchison J. Review of Progress with Bagasse for Use In Industry (A Review of Progress in Purchasing, Handling, Storage and Preservation of Bagasse) J.E. Proc. Intern. Soc. Sugar Cane Technologists, Vol.14:1202-1217, Franklin Press, Baton Rouge LA, USA. 1971.

Atchison J.E. Utilization of Bagasse for Manufacture of Pulp, Paper and Board. Indian Pulp and Paper, 17(1):37-46. 1962.

Cusi D.S. Conservación y almacenaje del bagazo. Sugar y azúcar. Septiembre, 75(9). 1980.

Chen S. Model-Free Kinetic Analysis of Thermal Decomposition of Sweet Sorghum Bagasse. 6th OAPS, Working Paper Series. Pp. 1-7. 2009.
García A. Cinética de la degradación del bagazo almacenado. Revista CENTRO AZUCAR, 16(2):101-109. Abril-junio,1989.

Hesch R. Method for Storing Bagasse. United States Patent 3964961.1976.

Huang H.C. A Study of Changes in Bagasse During Storage and the Effect on the Quality of Bagasse Particle Boards. On: Proceedings of the 15th congress of the International Society of Sugar Cane Technology. 3, pp. 1840-1850. 1974.

Hunter W.R. Bagasse Pulp Uses in Papermaking [en línea] HurterConsult Incorporated Canada, 2001. Disponible en: URL: http//:www.hurterconsult.com

Jiménez A.S. El bagazo como fibra alternativa. MARI-PAPEL, 5(5):17-23. Oct-nov, 1997. ISSN 0121-6554.

Kadam L.K. Environmental Life Cycle Implications of Using Bagasse-Derived Ethanol as a Gasoline Oxygenate in Mumbai (Bombay). National Energy Technology Laboratory Pittsburgh, Pennsylvania, USA and USAID, New Delhi, India 89 p. 2000.

Liese W. Deterioration of Bagasse During Storage and its Prevention [en línea]. Revista Floresta. 1978. Disponible en: http://ojs. c3sl.ufpr.br/ojs2/index.php/floresta/article/viewFile/6229/4444

Lois C.J.A. Almacenamiento de bagazo para su utilización industrial. Memorias del Seminario Internacional Generación Comercial de Energía Eléctrica en la Agroindustria Cañera, México. GEPLACEA, ASAZGUA, GTZ., pp.297-332. 1995.

Lois C.J.A. Sistemas y equipos de desmedulado en la industria del bagazo de caña de azúcar. ICIDCA. Edit. Científico-Técnica. La Habana Cuba. 1985. 9-20 pp.

Macaskill C. A Reaction-Diffusion Model of Stored Bagasse. ANZIAMJ, 43:13-34. 2001.

Mariani S.A. Secuencias de blanqueo ECF y TCF aplicadas a pulpa de bagazo de caña. Revista Forestal Venezolana, 49(1):27-31. 2005.

Molina R. Pulpa y papel de bagazo en los umbrales del nuevo siglo. En: Memorias del VII Congreso Internacional sobre Azúcar y Derivados de la Caña Diversificación 2002. La Habana, Cuba. 2002.

Rainey T.J., Clark N. An Overview of Bagasse as a Resource for the Australian Paper Industry. On: Hogarth, DM, Eds. Proceedings 2004, Conference of the Australian Society of Sugar Cane Technologists 26, Australia, Queensland, Brisbane. 2004.

Rainey T.J.G., Covey D.Sh. An Analysis of Australian Sugarcane Regions for Bagasse Paper Manufacture. International Sugar Journal, CVIII (1295): 640-644, November, 2006.

Rangan S.G., Rangamannar G. Development in Bagasse Fiber Preparation for the Manufacture of Fine Papers and Newsprinta Review of Facilities in Two Successful Indian Mills Tappi Pulping Conference. 1998.

Rangan S.G. Conservation of Bagasse During Storage. Indian Pulp and Paper IPPTA, 1(2):26-36. 1989. 
Rutiaga Q.J.G.. Anzaldo J.H., Vargas R.J.J. y Sanjuán D.R. Propiedades de resistencia de una pulpa kraft de pino mezclada con médula de bagazo de caña de azúcar. Madera y Bosques, 8(2):17-26. 2002.

Salaber J., Maza. Ritter Biological Treatment Process for Bagasse Bulk Storage. TAPPI Non-wood Plant Fiber Pulping Progress Report, No 2. 1971.

Sharkawy K, Rousu P, Haavisto S., Paulapuro H. Control of Bagasse Pulp Quality by Fractionation and Refining. Proceedings of the 60th Appita Annual Conference, Melbourne, Australia, pp.365-372. 2005.

Sharma R.K., Yadav K.R, Maheshwari V.L, Kothari R.M Baggasse Preservation: A Need for a Biotechnological Approach. Critical Reviews in Biotechnology, 20(4):237-263. 2000.

Sanjuán-Dueñas R. Obtención de pulpas y propiedades de las fibras para papel. Agata editores. México. 1997.
Savcor-Indufor Oy. China: Technical Assistance for the Sustainable Development of the Non-wood Pulp and Paper IndustryPulp Quality Comparison Technical Report - Module 6 Final Helsinki Finland, 18 p. 2007.

Schmidt O. Succession and Activity of Microorganisms in Stored Bagasse. Applied Microbiology and Biotechnology, 5(1):69-77. 1977.

TAPPI. Technical Association of the Pulp and Paper Industries. TAPPI Test Methods 2000-2001. Atlanta, Georgia, USA. 2000.

Triana O.L.M. Saavedra. Atlas del bagazo de caña de azúcar. GEPLACEA, PNUD, ICIDCA. 1990. Pp.37-45.

Yadav K.R., Rajesh-Sharma K., Kothari R.M. Preservation of Bagasse Through Biotech Approach for Pulp and Paper Industry. Indian J Chem Technol, 10(6):587-592. 2003.

Zegarra J. Paper From Bagasse. Nonwood Plant Fiber Pulping No. 8. TAPPI press. 1977. Pp. 3-8.

\section{Semblanza del autor}

Noé Aguilar-Rivera. Es ingeniero químico, maestro en ciencias de productos forestales y candidato a doctor en ciencias ambientales. Actualmente docente investigador de la Universidad Veracruzana, pertenece al cuerpo académico "Ciencia y tecnología de la caña de azúcar" con la línea de investigación biotecnología y derivados de la caña de azúcar. Ha trabajado en diversos proyectos académicos e industriales en el tratamiento y uso de residuos agroindustriales, celulosa y papel y caña de azúcar. 